\title{
A Novel Adaptive Algorithm for 3D Finite Element Analysis to Model Extracortical Bone Growth
}

Vee San Cheong ${ }^{1, *}$, Gordon W. Blunn ${ }^{1}$, Melanie J. Coathup ${ }^{1}$, Paul Fromme ${ }^{2}$

${ }^{1}$ John Scales Centre for Biomedical Engineering, Institute of Orthopaedics and

Musculoskeletal Science, University College London, Royal National Orthopaedics

Hospital, Stanmore, UK

${ }^{2}$ Department of Mechanical Engineering, University College London, London, UK

*Corresponding author

Email address: v.cheong@ucl.ac.uk 


\title{
A Novel Adaptive Algorithm for 3D Finite Element Analysis to Model Extracortical Bone Growth
}

\author{
Extracortical bone growth with osseointegration of bone onto the shaft of \\ massive bone tumour implants is an important clinical outcome for long-term \\ implant survival. A new computational algorithm combining geometrical shape \\ changes and bone adaptation in 3D Finite Element simulations has been \\ developed, using a soft tissue envelope mesh, a novel concept of \\ osteoconnectivity, and bone remodelling theory. The effects of varying the initial \\ tissue density, spatial influence function and time step were investigated. The \\ methodology demonstrated good correspondence to radiological results for a \\ segmental prosthesis.
}

Keywords: external bone remodelling, segmental prosthesis, osseointegration

\section{Introduction}

Bone cancer occurs primarily in children and adolescents during periods of rapid bone turnover or growth spurts. Young patients who receive segmental prostheses after the surgical removal of bone cancer require implants that last through their growth phase and into adult life, but aseptic loosening is the primary mode of implant failure (Unwin et al. 1996). Using finite element (FE) method to analyse the effects of implant on bone remodelling, the common approaches are to conduct static stress analysis at different stages of follow-up (Galloway et al. 2013, Fromme et al. 2017), model changes in the density of existing bone adjacent to an implant (Tomaszewski et al. 2012), the healing of the defect (gap) around the implant stem, or ingrowth of bone onto the surface of the implant stem, (Dickinson et al. 2012, Tarala et al. 2011). To verify results, clinical radiographs are often compared qualitatively with FE predictions, due to difficulties in validating the density of the bone (Tomaszewski et al. 2012, Dickinson et al. 2012). For massive segmental prostheses used in bone cancer the presence of extracortical bone 
formation and attachment onto the shaft of the implant has been demonstrated to improve the survivorship of these implants at 10 years, from $75 \%$ to $98 \%$ (Fig. 1) (Coathup et al. 2013). Unlike bone adaption adjacent to hip stems, which is associated with changes in bone density, the formation of extracortical bone involves bone growth over the shaft of the implant changing the bone shape. Modelling and predicting extracortical bone growth using FE could thus advance the design and assessment of implants.

\section{[Fig. 1 near here]}

Bone remodelling algorithms implemented in FEA can overcome the limitations of static FEA to evaluate the performance and failure risk of various implant designs as they simulate the changes in bone density in the presence of a prosthesis. One of the most commonly utilised algorithms is the adaptive elasticity theory (Cowin and Hegedus 1976; Cowin and Firoozbakhsh 1981), which was adapted for studying bone remodelling around implants using strain energy density (SED) as the stimulus (Huiskes et al. 1987, Weinans et al. 1992). Bone remodelling refers both to the internal adaptation of bone density (internal remodelling), and the displacement of surface nodes leading to a change in periosteal geometry (external or surface remodelling) in 2D and 3D (Hart et al. 1984, Huiskes et al. 1987). FE models for implant analysis have focussed on changes in internal density (Lin et al. 2009, Tarala et al. 2011, Tomaszewski et al. 2012), rather than geometrical shape changes. Instabilities introduced by the significant volume and shape changes make it challenging to employ surface remodelling to capture the process of additive bone formation in segmental prostheses.

Advances in remodelling theories have focussed on mitigating numerical stability and problem-dependent convergence (Harrigan and Hamilton 1992, Huiskes et al. 1987). This is observed visually as a checkerboard of discontinuities, which would 
violate the continuum assumption in FEA (Mullender et al. 1994). For the elementbased approach, stresses are averaged from each integration point to update the bone density at the centroid, causing discontinuities in both stresses and densities across the element boundaries. Both higher order elements and finer meshes reduce checker boarding, but the use of filtering with linear elements achieves similar results, while reducing computational cost (Li et al. 2001). A new method for updating bone density has been proposed (Jacobs et al. 1995). Node-based approaches ensure continuity by calculating the bone density at each node, averaging stress and strain. However, nodebased approaches require the computationally intensive calculation of the stiffness matrix at each integration point through extrapolation of the nodal values (Chen et al, 2007).

The influence of spatial functions has only been investigated in 2D and for internal remodelling (Marzban et al. 2012, Zhu et al. 2005). Backward Euler integration has been recommended as it provides a more stable solution (Harrigan and Hamilton 1993). However, most literature used the forward Euler method with a small constant time step, formulated as a fraction of the time span, so that it does not affect the final result, yet minimises computational time, or calculated empirically after a trial run to keep the initial change in modulus within 10-20\% (Huiskes et al. 1987, Li et al. 2007, Marzban, et al. 2012). The long computation time has led authors to propose extrapolation methods or raise the order of the remodelling equation, to accelerate the simulation process without losing accuracy in 2D (Marzban et al. 2012, Mohaghegh et al. 2014, Zhu et al. 2005).

Recent work in bone remodelling focussed on integrating bone adaption and tissue differentiation to model the process of healing better. In mechano-regulatory models of fracture healing, a callus is usually assumed and granulation tissue fills the 
space from an origin site through a diffusion process (Lacroix et al. 2002).

Differentiation rules based on the mechanical stimulus are applied to update the material properties and determine types of tissue formed (Huiskes et al. 1997, Prendergast 1997). These models assume a fixed predefined gap that would close and heal and use a mechanobiological differentiation algorithm. The development of extracortical bone adjacent to an implant is a different scenario as bone growth, which is largely intramembranous, occurs from bone at the transection site over the implant surface. Other advances in the numerical approach for bone adaption include anisotropic bone remodelling based on continuum damage mechanics (CDM). CDM proposes that the rate and direction of remodelling is based on the micro-damage accumulated (Predergast and Taylor, 1994, Doblare and Garcia, 2001). As this study focusses on extracortical bone formation, the adaptive elasticity theory was used. This paper presents an integrated computational framework combining stress analysis, bone remodelling and extracortical bone formation that can be used to evaluate implant designs and predict clinical outcome for bone cancer patients. It proposes a novel method of osteoconnectivity to model the additive nature of bone formation through the use of a soft tissue envelope of low initial modulus, by incorporating the sequential laying down of new bone next to existing bone stock (Prendergast 1997). A cylindrical model was used to illustrate the development of the enhanced computational algorithm, and determine the effects initial density, choice of averaging, and time steps have on the distribution, stability and accuracy of the result. The model was applied to predict extracortical bone growth around a full sized segmental prosthesis, and compared against radiographs of an implanted segmental prosthesis in a 2 years follow-up (Fromme et al. 2017). This is the first work to develop an adaptive model for extracortical bone growth around implants in 3D, accounting for the process of growth observed clinically. 
Moreover, this is the first time that time dependant extracortical bone formation has been modelled. This work investigated methods to improve the element-based approach for bone remodelling in 3D and introduced adaptive time stepping to improve the efficiency of the adaptive solution.

\section{Methodology}

\subsection{Enhanced 3D bone formation and adaptation model}

The integrated framework is based on the adaptive elasticity theory (Huiskes et al. 1987, Weinans et al. 1992) and a concept of connectivity to control extracortical bone formation in a soft tissue envelope mesh around the area of interest (Fig. 2).

Osteoconnectivity is introduced as a map of neighbouring elements that permits only the remodelling of elements that are adjacent to bone stock or elements that have remodelled in previous time steps.

[Fig. 2 near here]

The material property of each element $i$ is based on the mechanical stimulus $\mathrm{S}_{i}$ (Huiskes et al. 1987):

$$
S_{i}=\frac{U_{i}}{\rho_{i}}-k
$$

where $U$ is the remodelling signal and $\mathrm{k}$ is a reference signal value such that the stimulus is zero. The choice of remodelling signal type has been investigated previously and SED is used here as it has been found to agree well with clinical data (Schmitz et al. 2004). For simplicity, $k$ is assumed to be a single value, but it could vary according to location and density. Using the idea of a minimum inhibitory signal before remodelling occurs (Frost 1988), a bandwidth of $\rho$ is included to incorporate a lazy zone without remodelling: 


$$
\begin{array}{lr}
\mathrm{S}_{i}=\frac{U_{i}}{\rho_{i}}-(1+\delta) k & \text { if } S>(1+\delta) k \\
\mathrm{~S}_{i}=0 & \text { if }(1-\delta) k \leq S \leq(1+\delta) k \\
\mathrm{~S}_{i}=\frac{U_{i}}{\rho_{i}}-(1-\delta) k & \text { if } S<(1-\delta) k
\end{array}
$$

To reduce the checkerboard problem of discontinuities, a spatial influence function $f_{j}(x, y, z)$ that determines the contribution of other elements' signals to the element under consideration is included (Mullender et al. 1994). The remodelling rate, in terms of change in density $\rho$ of each element $i$ is thus regulated by its own stimulus value, relative to the signals of the neighbouring elements, and scaled by a time constant $B:$

$$
\frac{\partial \rho(i, t)}{\partial t}=B \sum_{j=1}^{N} f_{j}(x, y, z) S_{j}
$$

The Euler forward method is used iteratively to solve ordinary differential equations (ODE) to obtain the new density:

$$
\rho_{i}(t+\Delta t)=\rho_{i}(t)+\frac{\partial \rho(i, t)}{\partial t} \Delta t \quad 0<\rho \leq \rho_{c b}
$$

where $\Delta t$ is the time step and $\rho_{\mathrm{cb}}$ is the upper limit for the density of cortical bone. A mathematical relationship that relates density to elastic modulus, using constants $C$ and $\gamma$, is used to update the stiffness matrix for the next increment (Carter 1984):

$$
E(i, t+\Delta t)=C \rho(i, t+\Delta t)^{\gamma}
$$


Following literature, the parameters are set as $B=1.00 \mathrm{gcm}^{-3}, k=0.004 \mathrm{Jg}^{-1}, \delta$ $=10 \%, E_{\mathrm{cb}}=12 \mathrm{GPa}, C=3790 \mathrm{MPa}\left(\mathrm{gcm}^{-3}\right)^{-2}$ and $\gamma=3$ (Carter 1984, Weinans et al. 1992).

\subsection{Effect of averaging}

Two spatial influence functions were assessed for their efficacy to resolve the checkerboard problem (Jacobs et al. 1995, Mullender et al. 1994). Standard averaging was carried out by averaging the element in question and its nearest neighbours. This method is similar to the node-based approach for bone remodelling which reduces discontinuities with good results (Jacobs et al. 1995). Weighted averaging (50\% element, 50\% average neighbours) was derived from the method proposed by Mullender et al. (1994), but covers a smaller volume (only neighbouring elements) to reduce the amount of smoothing.

\subsection{Time step}

To ensure stable simulations and minimise computation time, adaptive time stepping was employed and compared to results using fixed time steps ( 1 and 0.5$)$. The RungeKutta Midpoint Method was used to approximate the time dependent FE solutions for Eq. 3. The difference between error estimate $\varepsilon$ and the target error $\varepsilon 0$ was used to modify the step size with a safety factor of $\beta=0.9 . \varepsilon_{0}$ was varied at $1,0.1$ and $0.01 \%$. This was tested for a uniform, cylindrical model using initial densities of $0.8 \mathrm{gcm}^{-3}$, $0.5 \mathrm{gcm}^{-3}$ and $0.297 \mathrm{gcm}^{-3}(100 \mathrm{MPa})$ to cover a range of values used in literature (Dickinson et al. 2012, Huiskes et al. 1987, Weinans et al. 1993). Predicted tissue densities were compared against the analytical solution, obtained by solving the ODE numerically using the stricter Midpoint method, with a time step of 0.001 to keep the truncation error below $0.1 \%$. 


\subsection{Parametric studies on connectivity for extracortical bone formation}

To understand the influence of connectivity on the distribution of tissue density and the accuracy of results, parametric studies for initial density, time step, threshold value, and averaging functions were conducted. Initial density was varied as in Section 2.3, but only the case for $0.297 \mathrm{gcm}^{-3}$ is reported as the results were similar. Fixed time steps of 1E-4 or 1E-3 were used until all elements had started remodelling. Adaptive stepping using $\varepsilon_{0}$ of $0.01 \%$ was used once all the elements had started remodelling, according to section 2.3.

\subsection{Cylindrical model}

The algorithms were tested using a uniform, cylindrical model of radius $5 \mathrm{~mm}$ and height 10mm, with 48000 first-order strain-smoothed tetrahedral elements of size $0.5 \mathrm{~mm}$. Linear elements with a fine mesh around the grooved collar were used (Section 2.6), as results for linear and higher order elements converge with increasing mesh density (Dumont et al. 2005; Bright and Rayfield 2011). Strain-smoothed elements with improved accuracy in bending and torsion were employed (Nguyen-Thoi et al. 2010).

The FEA was conducted in Marc 2015.0 (MSC Software Corporation, Santa Ana, USA) using custom-written subroutines. Two meshing algorithms available in the FE package were used to assess the mesh quality. IsoMesh generated a regular stacked structure throughout, but TetMesh yielded 2 different tetrahedral patterns; in the core and the outer region. The distal midpoint node was fully constrained and all other nodes on the distal face were restricted from axial movement. A constant uniaxial pressure of $2.4 \mathrm{kN}$ was applied proximally. A Poisson's ratio of $v=0.3$ and isotropic material formulation using Eq. 5 were used. 


\subsection{Application to segmental prosthesis}

A distally implanted cemented femoral prosthesis was considered and the middle $50 \%$ of the femur was modelled as a cylinder $180 \mathrm{~mm}$ long with a cortical thickness of 4.5mm (Fig. 3), based on literature values (Gosman et al. 2013). The Stanmore modular prosthesis was modelled with a $12 \mathrm{~mm}$ stem and a $0.75^{\circ}$ taper (Taylor and Walker 2001). The length of the stem was $130 \mathrm{~mm}$ long, similar to other brands of prosthesis (Tomaszewski et al. 2012). A 1x1x1 mm grooved, 18mm tall collar was modelled as one piece with the stem. A $1 \mathrm{~mm}$ gap separates the bone/cement and the distal end of the collar as observed in radiographical images (Fromme et al. 2017). The soft tissue envelope was modelled as $40 \mathrm{~mm}$ tall, with a diameter of $42 \mathrm{~mm}$ and $45^{\circ}$ and $59^{\circ}$ chamfer at the proximal and distal ends respectively.

\section{[Fig. 3 near here]}

The model was meshed with element lengths of $0.4 \mathrm{~mm}$ and $1 \mathrm{~mm}$ for the soft tissue and cement respectively. The bone and implant were meshed with a size of $2 \mathrm{~mm}$. Mesh refinement was conducted at the tip of the stem and collar to give an average size of $0.5 \mathrm{~mm}$. This yielded 1,193,439 first-order tetrahedral elements in the model. Mesh refinement studies were conducted with a focus on the contact interfaces and locations of stress concentration. Increasing the mesh from 1.2 million to 4.8 million elements led to less than 3\% change in the von Mises stress around the soft tissue.

Isotropic material properties were used for the Ti-6Al-4V implant $(\mathrm{E}=110 \mathrm{GPa}, v$ $=0.3)$, cortical bone $(\mathrm{E}=12 \mathrm{GPa}, v=0.3)$ and PMMA cement $(1.8 \mathrm{GPa}, v=0.4)$. Soft tissue elements have inhomogeneous isotropic material properties as per Eq. 5. Remodelling was simulated in the soft tissue envelope, but no remodelling or density change was permitted in the existing bone. A friction coefficient of 0.3 was assigned to the metal-cement and metal-soft tissue interfaces (Shirazi-Adl et al. 1993), while all 
other interfaces were assumed to be perfectly bonded. The model was fully constrained proximally, and axial load, bending moment and torsion, corresponding to the physiological walking load for a $75 \mathrm{~kg}$ person, were applied at the distal face of the implant (Fig.3) (Table 1) (Taylor and Walker 2001).

\section{[Table 1 near here]}

\section{Results}

\subsection{Effect of mesh quality and time step}

The cylindrical models were used to investigate the effect of the mesh quality and time stepping on the uniformity of the time-dependant remodelling. The different tetrahedral arrangements using TetMesh and IsoMesh resulted in worst element geometry aspect ratios of 7.0 and 5.5 respectively. For the first time increment of $1 \mathrm{E}-3$, the maximum deviation from analytical values was $6.7 \times 10^{-5} \%$ for the TetMesh model, increasing to $6.1 \%$ at equilibrium. The IsoMesh model showed no deviation for all time steps. The average tissue densities were respectively $0.56 \%$ and $0.012 \%$ higher than the analytical values.

The choice of time step and initial density affected the type of remodelling curve, shown in Fig. 4 for an initial modulus of 100MPa (density $0.297 \mathrm{gcm}^{-3}$ ). Using a fixed time step of 1.0 with initial densities of 0.5 or $0.8 \mathrm{gcm}^{-3}$ produced a hyperbolic curve, agreeing with the analytical solution. However, using an initial modulus of 100MPa caused the density to overshoot to $1.274 \mathrm{gcm}^{-3}$ in the first time step (Fig. 4 inset). Reducing the fixed time step to 0.5 still caused an overshoot (albeit smaller), with an error at $\mathrm{t}=10$ of $10.9 \%$ from the analytical result. The system was observed to recover to yield an error of $0.00174 \%$ at equilibrium (Table 2). Using adaptive time stepping with target errors of $0.1 \%$ and $0.01 \%$ for the $100 \mathrm{MPa}$ case, the deviations at 
$\mathrm{t}=10$ were $0.948 \%$ and $0.325 \%$ respectively and below $0.02 \%$ at equilibrium. Adaptive stepping gave a computational time saving of more than $75 \%$ for all tested initial densities.

\section{[Fig. 4 near here]}

[Table 2 near here]

\subsection{Connectivity}

The inclusion of connectivity only allows successive layers of elements to remodel and causes a slight delay and change in the response curve for the first few increments (Fig. 5 inset). The connectivity and the analytical average tissue density curves are very similar. Within each initial time step, the results were very similar, so only the case for no averaging is shown. The connectivity cases using initial stepping had a deviation of $1 \%$ at equilibrium. Using an initial step of $1 \mathrm{E}-4$ reduced the deviation to less than $0.017 \%$.

\section{[Fig. 5 near here]}

For the first time step, the stress field in the cylinder was uniform, causing the first (bottom) layer of elements to remodel uniformly. For the following time steps, the remodelled layers are slightly stiffer, restricting the radial movement of the outer elements in the layers above slightly. This causes higher SED at the edge, leading to a positive feedback loop such that the outer elements become stiffer than the inner elements. As subsequent layers remodel, the elements at the circumference become stiffer with a core of elements of lower density inside the cylinder. This effect diminished towards the top layer(s), where the stresses are relatively uniform.

The final density distribution is shown in Fig. 6 for two different initial time steps and local averaging. For the initial time step of 1E-3, standard and weighted averaging reduced the maximum deviation at equilibrium from $15.3 \%$ to $4.8 \%$ and $6.8 \%$ 
respectively (Fig. 6a). Averaging also reduced the checkerboard problem, which is more pronounced for tetrahedral elements. Reducing the time step decreases the initial density changes and thus non-uniformity of the remodelling. For the smaller initial time step of $1 \mathrm{E}-4$, the maximum deviations at equilibrium were reduced to $1.5 \%, 0.5 \%$, and $0.7 \%$ for no, standard, and weighted averaging respectively.

[Fig. 6 near here]

\subsection{Segmental prosthesis model}

Fig 7 compares the extracortical bone formation around the grooved implant collar predicted by the two algorithms, against radiological results available at 3 different time points. Immediately post-surgery, only the resected bone remains (Fig. $7 \mathrm{a}$ ). At $\mathrm{t}=0.2$, the non-connectivity model predicts remodeling throughout the height of the collar (Fig. 7b), while the connectivity model restricted remodeling to the region below the first groove (Fig. 7c). At $\mathrm{t}=4$, the connectivity model predicted the growth of a pedicle that extends radially outward. This agrees well with the radiograph for 1 year follow-up, as there was limited ingrowth in the lower grooves and no bone growth at the top of the collar. At equilibrium, remodeled bone is predicted at the top of the grooved collar in the non-connectivity model, which is not observed clinically (2 year follow-up). The connectivity model predicted an increase in stiffness and larger extra-cortical bone formation on the medial side than the lateral side, which agrees well with the radiographs from long-term follow up (Figs 1, 7A). There were only 3 radiographs available from the clinical follow-up and exact time correspondence between computer time unit and periods of follow-up has not been determined (e.g. $\mathrm{t}=4$ and 150). The average tissue density at equilibrium for all remodelled elements was $0.075 \%$ lower for the connectivity model compared to the non-connectivity one. 


\section{Discussion}

The purpose of this paper was to develop a novel integrated bone remodelling algorithm model using adaptive FE simulations to predict extracortical bone growth over the shaft of an implant used to treat cancer patients. This novel approach used the adaptive elasticity theory (Huiskes et al. 1987, Weinans et al. 1992) for density changes and a concept of osteoconnectivity to enforce sequential bone formation starting at existing bone.

Initially a uniform cylindrical model with a uniaxial load was chosen, as the remodelling solution is stable (Harrigan and Hamilton, 1993). The average tissue density from FEA matched the expected analytical results, but the density distribution varied, depending on the mesh and time steps chosen. For a non-uniform mesh (TetMesh), small deviations at the initial steps increased significantly with time. This demonstrated the sensitivity of bone remodelling algorithms due to the presence of a positive feedback loop. Therefore, the use of meshes with appropriate time steps or error criteria is necessary to keep the deviation within acceptable range. The use of adaptive stepping is advantageous as it can match the remodelling rate, with initially large density changes and a long time to reach convergence. Time gains are achieved by keeping the initial time step small and increasing it near equilibrium. Using a suitable target error with connectivity, the continuum assumption for FEA can be maintained.

In the connectivity model, the initially uniform stress field increases at the outer elements and decreases at the core when the first layer begins to remodel. Due to the positive feedback loop, initial deviations propagate and lead to a higher density of the elements at the outer circumference. Using a smaller time step or averaging reduces the deviations at equilibrium. The average tissue density obtained using the two initial time steps were similar (Fig. 5). Reducing the time step both reduced the deviation of the 
average tissue density and the deviation of values at equilibrium (Fig. 6). This suggests that the choice of time step is critical in ensuring the solutions obtained are accurate. Some checkerboard problems were observed, similar to literature findings, as bone adaption algorithms are susceptible to the problems of discontinuities (Jacobs, et al. 1995, Mullender, et al. 1994). Both averaging and using a smaller time step have the effect of keeping the tissue density variation between elements small, for the continuum assumption in FEA to remain valid. The spatial influence functions used in this paper are light filtering methods that involved only neighbouring elements. Therefore, their ability to reduce discontinuities is expected to be less effective than that of the time step.

The segmental prosthesis model uses a large soft tissue envelope to model extracortical bone growth. A low initial density of $0.5 \mathrm{gcm}^{-3}$ was chosen, following histological results observing a combination of soft tissue and bone (Coathup et al. 2013), rather than higher values often chosen for implant analysis, corresponding to bone (Huiskes et al, 1987). Validation, e.g. through quantification of bone density (e.g. using DXA) is difficult, as bone cancer patients receive customized segmental prosthesis and due to imaging artefacts caused by the metallic implant. Therefore, the simulated bone formation and remodelling patterns were verified by visual comparison with clinical results for different remodelling stages, an acceptable approach used in implant analysis (Tomaszewski et al. 2012, Dickinson et al. 2012). An artefact of remodelling at the proximal end of the soft tissue envelope (not observed clinically), was due to higher stresses in the tissue chamfer. The final results obtained with or without connectivity were similar as they both converged towards a global equilibrium, but connectivity ensures that bone growth only occurred at physiologically relevant locations. Connectivity prevented bone formation from initiating at the distal end of the collar and predicted more remodelling on the medial 
side of the collar at equilibrium, corresponding well with the follow-up radiographs (Fig. 7). The FE models underestimated the extent of bone growth, as the pedicle reached about half the height of the collar in the radiograph, also observed in retrieval results (Coathup et al. 2013). Bone bridging over the collar was observed with little bone growth into the grooves (Fig. 7c). This is due to stress-shielding within the grooves. Therefore, clinical observations of bone growth into the grooves suggest that other biochemical factors and interface conditions should be considered, which may be associated with the use of osteoconductive coating.

This work showed that the integrated bone adaptation and extracortical bone formation algorithm is sensitive to changes in stress distribution when the elementbased approach is used. The use of a good quality mesh, with small initial time steps and averaging, helps to minimize discontinuities. The use of the Runge-Kutta Midpoint method allows for adaptive time stepping, retaining accuracy while reducing computational cost. The segmental prosthesis model used a soft tissue envelope to model and overcome problems associated with the large 3D geometrical shape. The process of additive bone formation was modelled, with good correspondence to clinical follow-up results. The enhanced remodelling algorithm has the potential to be developed further for other biomedical applications. With the development of new and porous implants through additive manufacturing technology, this integrated algorithm can be used for predicting additive bone formation into these scaffolds.

\section{Acknowledgements}

This work was funded by Orthopaedic Research UK (grant \#515). 


\section{References}

Bright JA, Rayfield EJ. 2011. The Response of Cranial Biomechanical Finite Element Models to Variations in Mesh Density. Anat Rec. 294:610-620.

Carter D. 1984. Mechanical loading histories and cortical bone remodeling. Calcif Tissue Int. 36:S19-S24.

Chen G, Pettet G, Pearcy M, McElwain DLS. 2007. Comparison of two numerical approaches for bone remodelling. Med Eng Phys. 29:134-139.

Coathup MJ, Batta V, Pollock RC, Aston WJ, Cannon SR, Skinner JA, Briggs TW, Unwin PS, Blunn GW. 2013. Long-term survival of cemented distal femoral endoprostheses with a hydroxyapatite-coated collar: a histological study and a radiographic follow-up. J Bone Joint Surg Am. 95:1569-1575.

Cowin SC, Hegedus D. 1976. Bone remodeling I: theory of adaptive elasticity. J Elast. 6(3):313-326.

Cowin SC, Firoozbakhsh K. 1981. Bone remodeling of diaphysial surfaces under constant load: Theoretical predictions. J Biomech. 14:471-484.

Dickinson A, Taylor A, Browne M. 2012. Implant-bone interface healing and adaptation in resurfacing hip replacement. Comput Methods Biomech Biomed Engin. 15:935-947.

Doblaré M, Garcia J. 2002. Anisotropic bone remodelling model based on a continuum damage-repair theory. J Biomech. 35:1-17.

Dumont ER, Piccirillo J, Grosse IR. 2005. Finite-element analysis of biting behavior and bone stress in the facial skeletons of bats. Anat Rec A Discov Mol Cell Evol Biol. 283A:319-330.

Fromme P, Blunn GW, Aston WJ, Abdoola T, Koris J, Coathup MJ. 2017. The Effect of Bone Growth onto Massive Prostheses Collars in Protecting the Implant from Fracture. Med Eng Phys. 41:19-25.

Frost HM. 1988. Vital biomechanics: Proposed general concepts for skeletal adaptations to mechanical usage. Calcif Tissue Int. 42:145-156.

Galloway F, Kahnt M, Ramm H, Worsley P, Zachow S, Nair P, Taylor M (2013). A large scale finite element study of a cementless osseointegrated tibial tray. J Biomech. 46:1900-1906.

Gosman JH, Hubbell ZR, Shaw CN, Ryan TM. 2013. Development of Cortical Bone Geometry in the Human Femoral and Tibial Diaphysis. Anat Rec. 296:774-787. 
Harrigan TP, Hamilton JJ. 1992. An analytical and numerical study of the stability of bone remodelling theories: Dependence on microstructural stimulus. J Biomech. 25:477-488.

Harrigan TP, Hamilton JJ. 1993. Finite element simulation of adaptive bone remodelling: A stability criterion and a time stepping method. Int J Numer Methods Eng. 36:837-854.

Hart RT, Davy D, Heiple K. 1984. A computational method for stress analysis of adaptive elastic materials with a view toward applications in strain-induced bone remodeling. J Biomech Eng. 106:342-350.

Huiskes R, Driel WDV, Prendergast PJ, Søballe K. 1997. A biomechanical regulatory model for periprosthetic fibrous-tissue differentiation. J Mater Sci Mater Med. 8:785-788.

Huiskes R, Weinans H, Grootenboer H, Dalstra M, Fudala B, Slooff T. 1987. Adaptive bone-remodeling theory applied to prosthetic-design analysis. J Biomech. 20:1135-1150.

Jacobs CR, Levenston ME, Beaupré GS, Simo JC, Carter DR. 1995. Numerical instabilities in bone remodeling simulations: the advantages of a node-based finite element approach. J Biomech. 28:449-459.

Lacroix D, Prendergast PJ, Li G, Marsh D. 2002. Biomechanical model to simulate tissue differentiation and bone regeneration: application to fracture healing. Med Biol Eng Comput. 40:14-21.

Li J, Li H, Shi L, Fok AS, Ucer C, Devlin H, Horner K, Silikas N. 2007. A mathematical model for simulating the bone remodeling process under mechanical stimulus. Dent Mater. 23:1073-1078.

Li Q, Steven GP, Xie YM. 2001. A simple checkerboard suppression algorithm for evolutionary structural optimization. Struct Multidisc Optim. 22:230-239.

Lin D, Li Q, Li W, Swain M. 2009. Dental implant induced bone remodeling and associated algorithms. J Mech Behav Biomed Mater. 2:410-432.

Marzban A, Canavan P, Warner G, Vaziri A, Nayeb-Hashemi H. 2012. Parametric investigation of load-induced structure remodeling in the proximal femur. Proc Inst Mech Eng H. 226:450-460.

Mohaghegh K, Pérez MA, García-Aznar JM. 2014. Accelerating numerical simulations of strain-adaptive bone remodeling predictions. Comput Methods Appl Mech Eng. 273:255-272. 
Mullender M, Huiskes R, Weinans H. 1994. A physiological approach to the simulation of bone remodeling as a self-organizational control process. J Biomech. 27:1389-1394.

Nguyen-Thoi T, Vu-Do HC, Rabczuk T, Nguyen-Xuan H. 2010. A node-based smoothed finite element method (NS-FEM) for upper bound solution to viscoelastoplastic analyses of solids using triangular and tetrahedral meshes. Comput Methods Applied Mech Eng. 199:3005-3027.

Prendergast PJ, Taylor D. 1994. Prediction of bone adaptation using damage accumulation. J Biomech. 27:1067-1076.

Prendergast PJ. 1997. Finite element models in tissue mechanics and orthopaedic implant design. Clinical Biomech. 12:343-366.

Schmitz M, Clift S, Taylor W, Hertig D, Warner M, Ploeg H, Bereiter H. 2004. Investigating the effect of remodelling signal type on the finite element based predictions of bone remodelling around the thrust plate prosthesis: a patientspecific comparison. Proc Inst Mech Eng H. 218:417-424.

Shirazi-Adl A, Dammak M, Paiement G. 1993. Experimental determination of friction characteristics at the trabecular bone/porous-coated metal interface in cementless implants. J Biomed Mater Res. 27:167-175.

Tarala M, Janssen D, Verdonschot N. 2011. Balancing incompatible endoprosthetic design goals: a combined ingrowth and bone remodeling simulation. Med Eng Phys. 33:374-380.

Taylor SJG, Walker PS. 2001. Forces and moments telemetered from two distal femoral replacements during various activities. J Biomech. 34:839-848.

Tomaszewski PK, Verdonschot N, Bulstra SK, Rietman JS, Verkerke GJ. 2012. Simulated bone remodeling around two types of osseointegrated implants for direct fixation of upper-leg prostheses. J Mech Behav Biomed Mater. 15:167175.

Unwin P, Cannon S, Grimer R, Kemp H, Sneath R, Walker P. 1996. Aseptic loosening in cemented custom-made prosthetic replacements for bone tumours of the lower limb. J Bone Joint Surg Br. 78:5-13.

Weinans H, Huiskes R, Grootenboer H. 1992. The behavior of adaptive boneremodeling simulation models. J Biomech. 25:1425-1441.

Weinans H, Huiskes R, Van Rietbergen B, Sumner D, Turner T, Galante J. 1993. Adaptive bone remodeling around bonded noncemented total hip arthroplasty: a 
comparison between animal experiments and computer simulation. J Orthop Res. 11:500-513.

Zhu X, Gong H, Gao B. 2005. The application of topology optimization on the quantitative description of the external shape of bone structure. J Biomech. 38:1612-1620. 
Table 1: Walking loads applied at distal end (implant) of the FE model, based on Taylor and Walker (2001).

\begin{tabular}{|llll|}
\hline Axial load/N & $\begin{array}{l}\text { Anterior- } \\
\text { Posterior bending } \\
\text { /Nm }\end{array}$ & $\begin{array}{l}\text { Medial-Lateral } \\
\text { bending/Nm }\end{array}$ & Torsion/Nm \\
\hline 2428 & 42.0 & 70.6 & 4.4 \\
\hline
\end{tabular}


Table 2: Average remodelled bone density $\left(\mathrm{gcm}^{-3}\right)$ for adaptive and fixed time stepping with relative difference to analytical solution for different time steps $(\mathrm{t}=10,100,500)$; number of time steps to $\mathrm{t}=500$ (equilibrium); cylindrical, uniform model, initial modulus: $100 \mathrm{MPa}$.

\begin{tabular}{|c|c|c|c|c|c|c|c|}
\hline Case & $t=10$ & Diff/\% & $t=100$ & Diff/\% & $\mathrm{t}=\mathbf{5 0 0}$ & Diff/\% & Steps \\
\hline $\begin{array}{l}\text { Fixed } \\
1.0\end{array}$ & 1.268 & 57.7 & 1.231 & 11.7 & 1.210 & 5.17 & 500 \\
\hline $\begin{array}{l}\text { Fixed } \\
0.5\end{array}$ & 0.892 & 10.9 & 1.108 & 0.631 & 1.150 & 0.00174 & 1000 \\
\hline $\begin{array}{l}\text { Adaptive } \\
1 \%\end{array}$ & 0.823 & 2.45 & 1.119 & 1.60 & 1.151 & 0.0626 & 27 \\
\hline $\begin{array}{l}\text { Adaptive } \\
0.1 \%\end{array}$ & 0.811 & 0.948 & 1.108 & 0.627 & 1.150 & 0.0130 & 69 \\
\hline $\begin{array}{l}\text { Adaptive } \\
0.01 \%\end{array}$ & 0.806 & 0.325 & 1.104 & 0.219 & 1.150 & 0.00609 & 197 \\
\hline $\begin{array}{l}\text { Analytical } \\
(1 \mathrm{e}-3)\end{array}$ & 0.804 & & 1.101 & & 1.150 & & \\
\hline
\end{tabular}




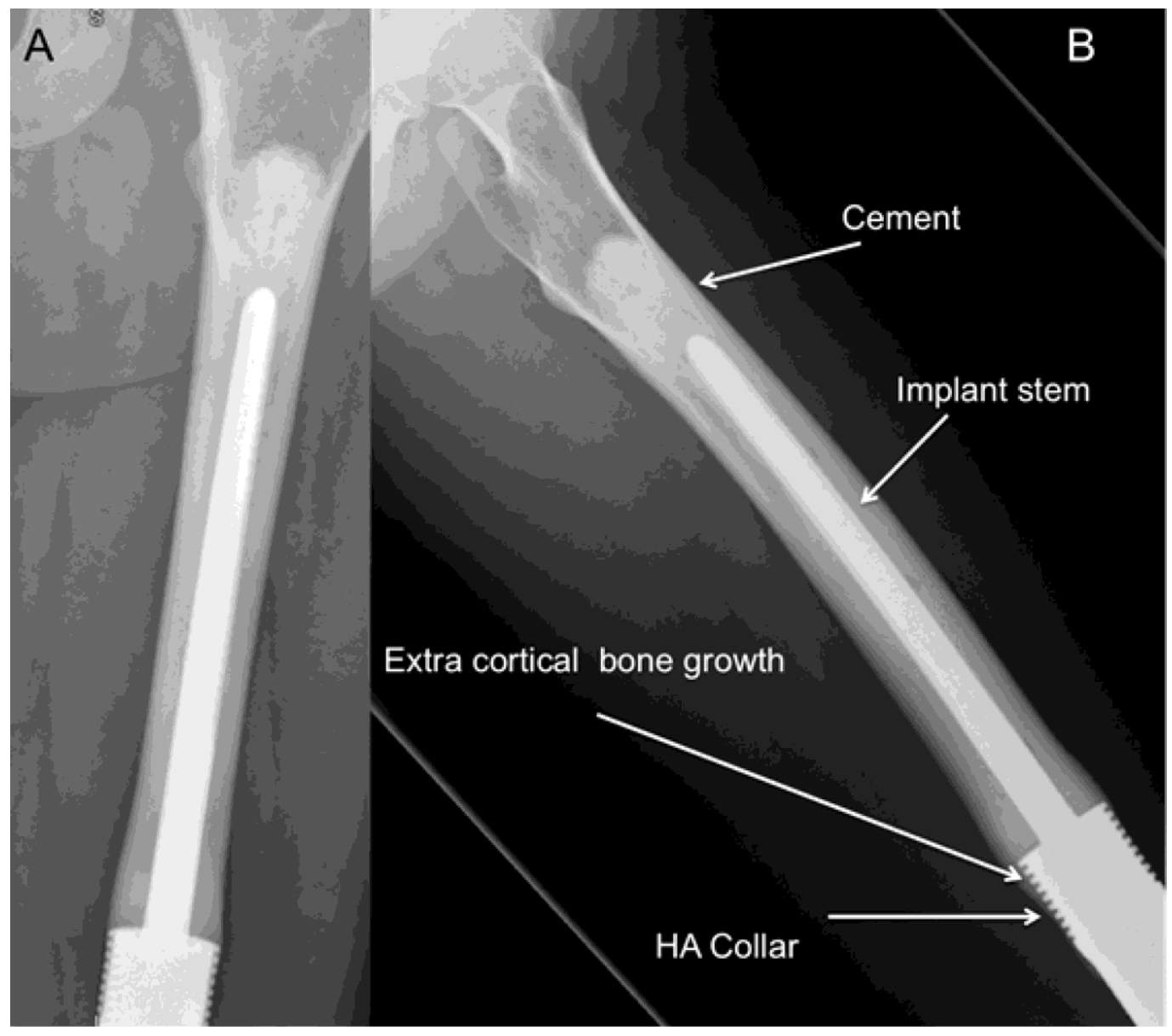

Fig. 1: Radiological images taken 8 years after distal femoral prosthesis insertion, showing hydroxyapatite (HA) coated collar with osseointegration. A: anterior radiograph; B: lateral radiograph.

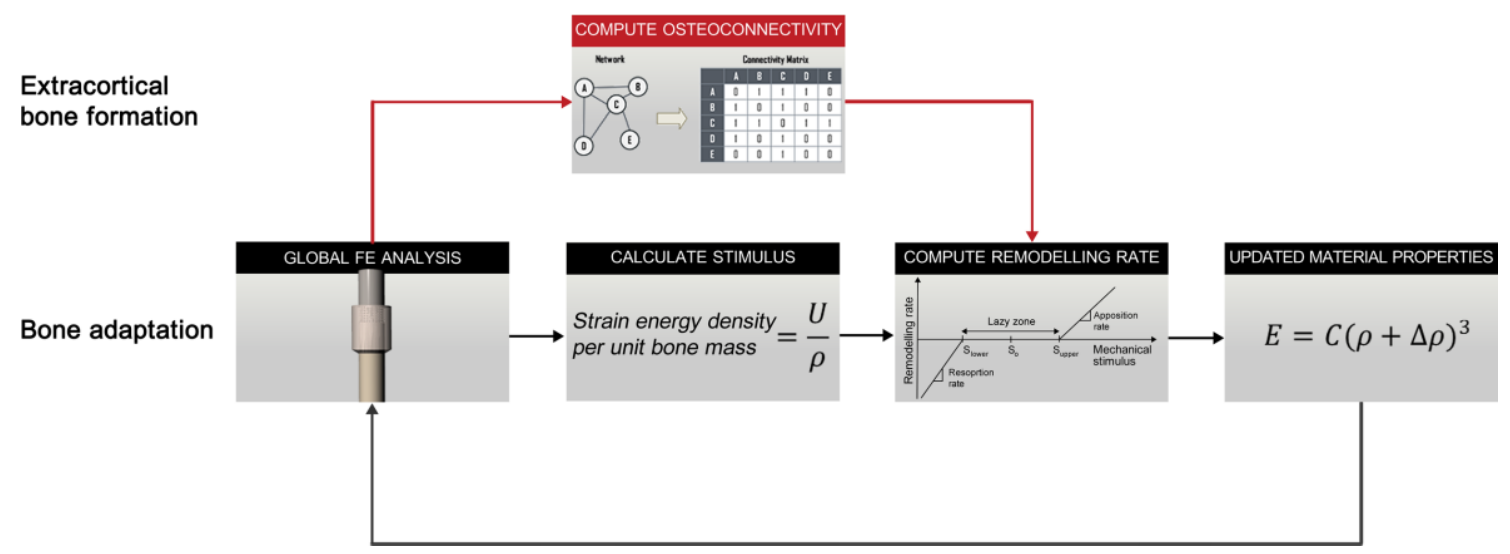

Fig. 2: Enhanced 3D bone formation and adaptation algorithm using osteoconnectivity matrix (top loop) with adaptive elasticity model (lower loop). 
A

B

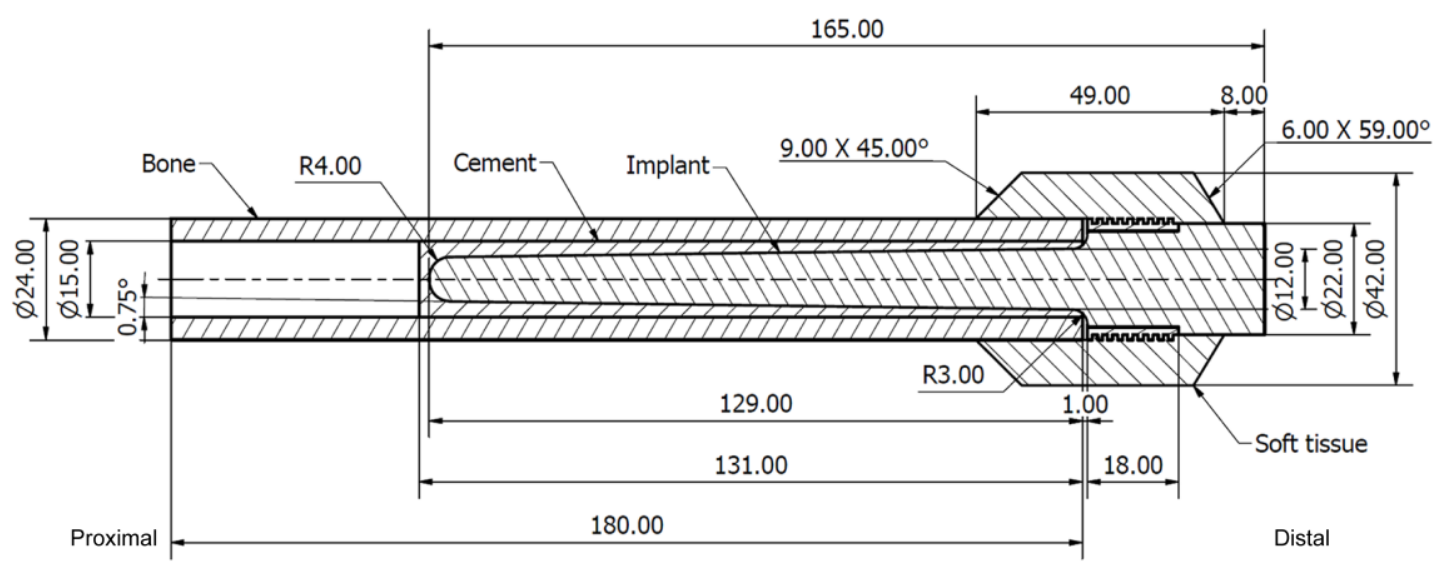

C

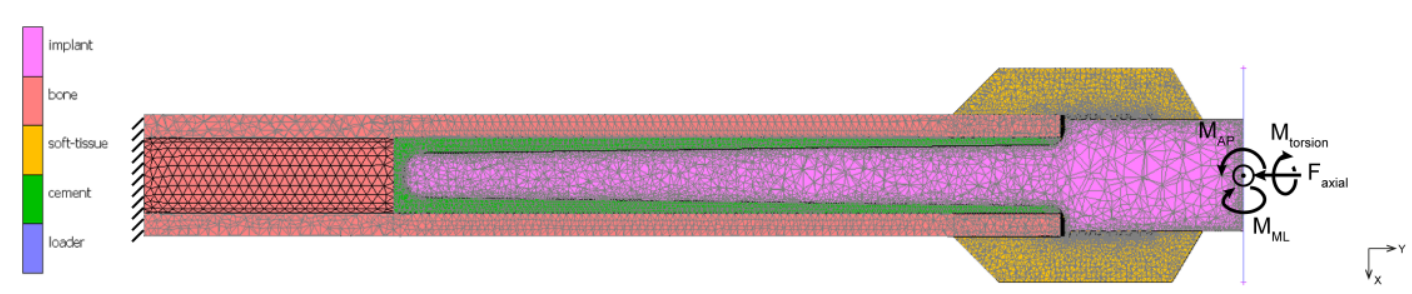

Fig. 3: (A) Segmental prosthesis implant with $1 \mathrm{~mm}$ grooved collar; (B) Geometry of implanted femur model, surrounded by soft tissue scaffold to model bone growth (all length in [mm]); (C) Materials and FE mesh. Arrow indicates load application.

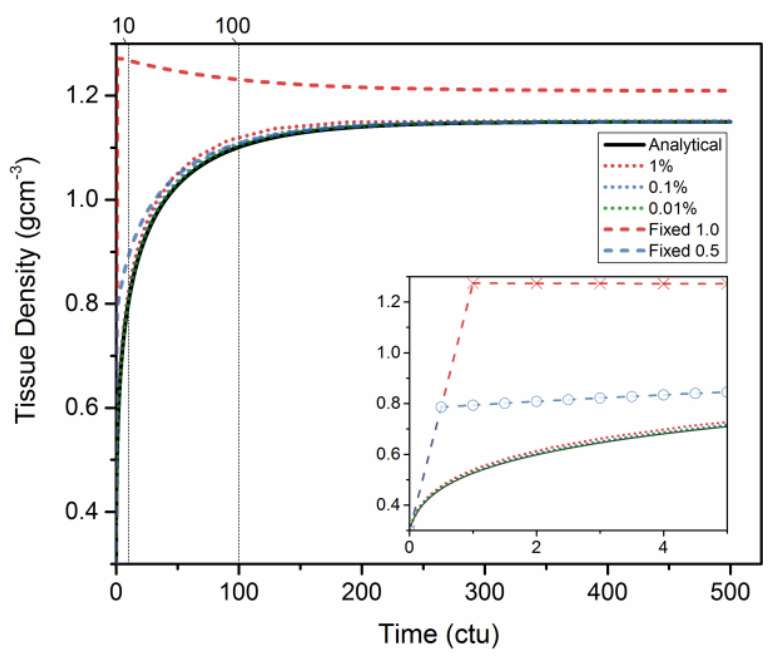

Fig. 4: Effect of time step on bone remodelling for uniform, cylindrical model (initial modulus: 100MPa, IsoMesh) with uniaxial load of $2.4 \mathrm{kN}$. Inset: zoom on initial steps. 


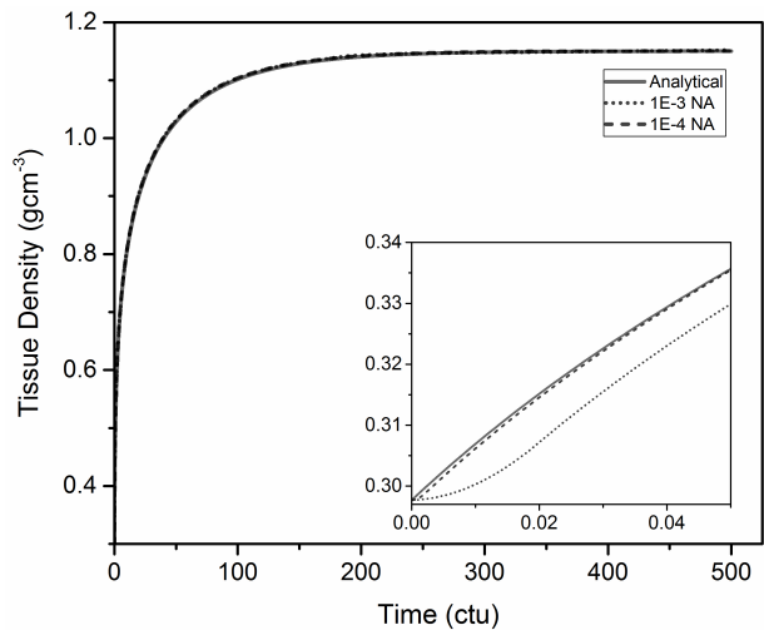

Fig. 5: Remodelling response curves (average density) of cylindrical model (initial modulus: $100 \mathrm{MPa}$, IsoMesh) with and without connectivity, compared to analytical solution (no connectivity), initial time stepping: 1E-3 and 1E-4. NA: no averaging.

A
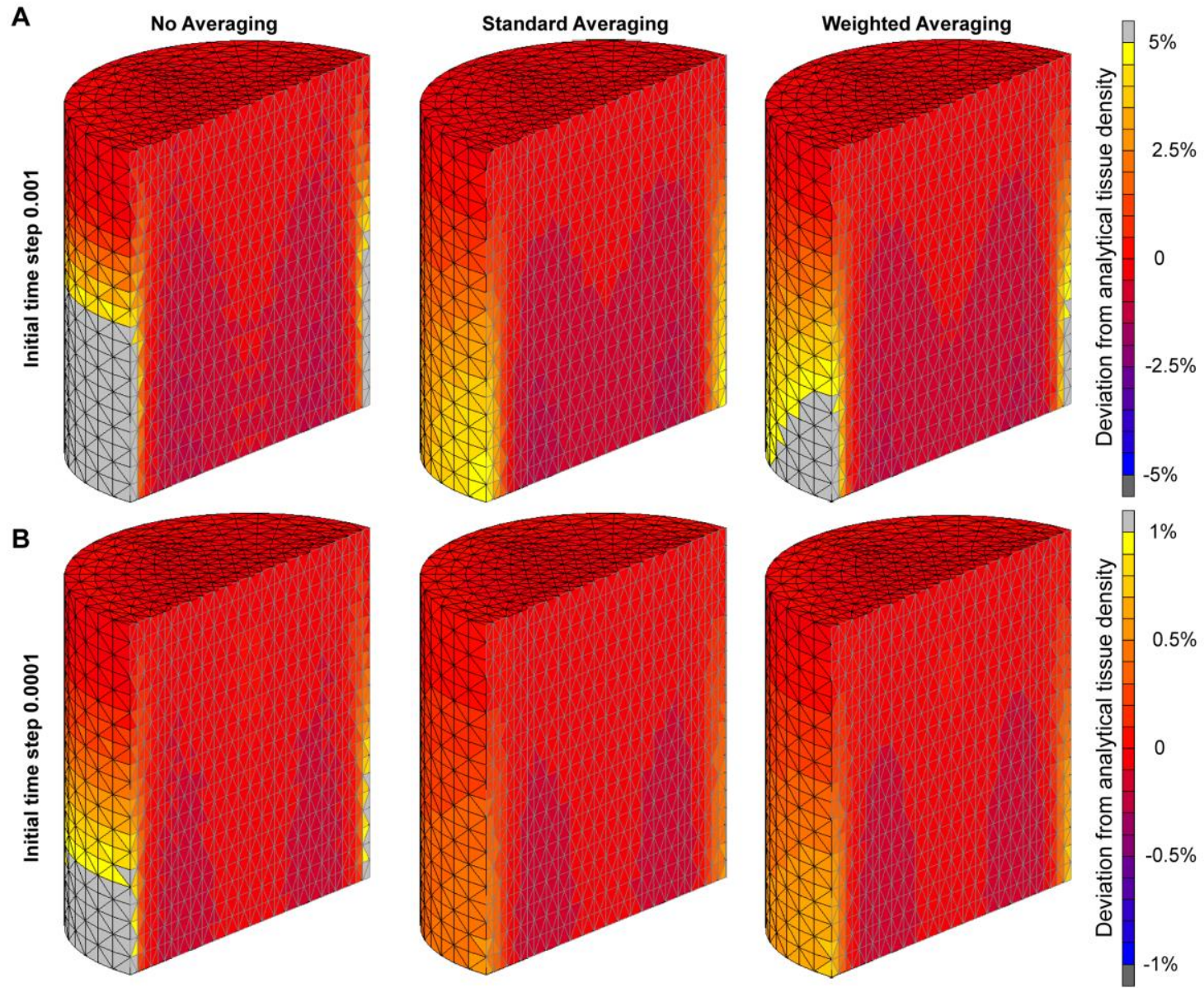

Fig. 6: Effect of initial time step and averaging on tissue density distribution at equilibrium ( $\mathrm{t}=500)$ of cylindrical model (initial modulus: 100MPa, IsoMesh), using connectivity; (A): Initial time step: 1E-3; (B): Initial time step: 1E-4. 

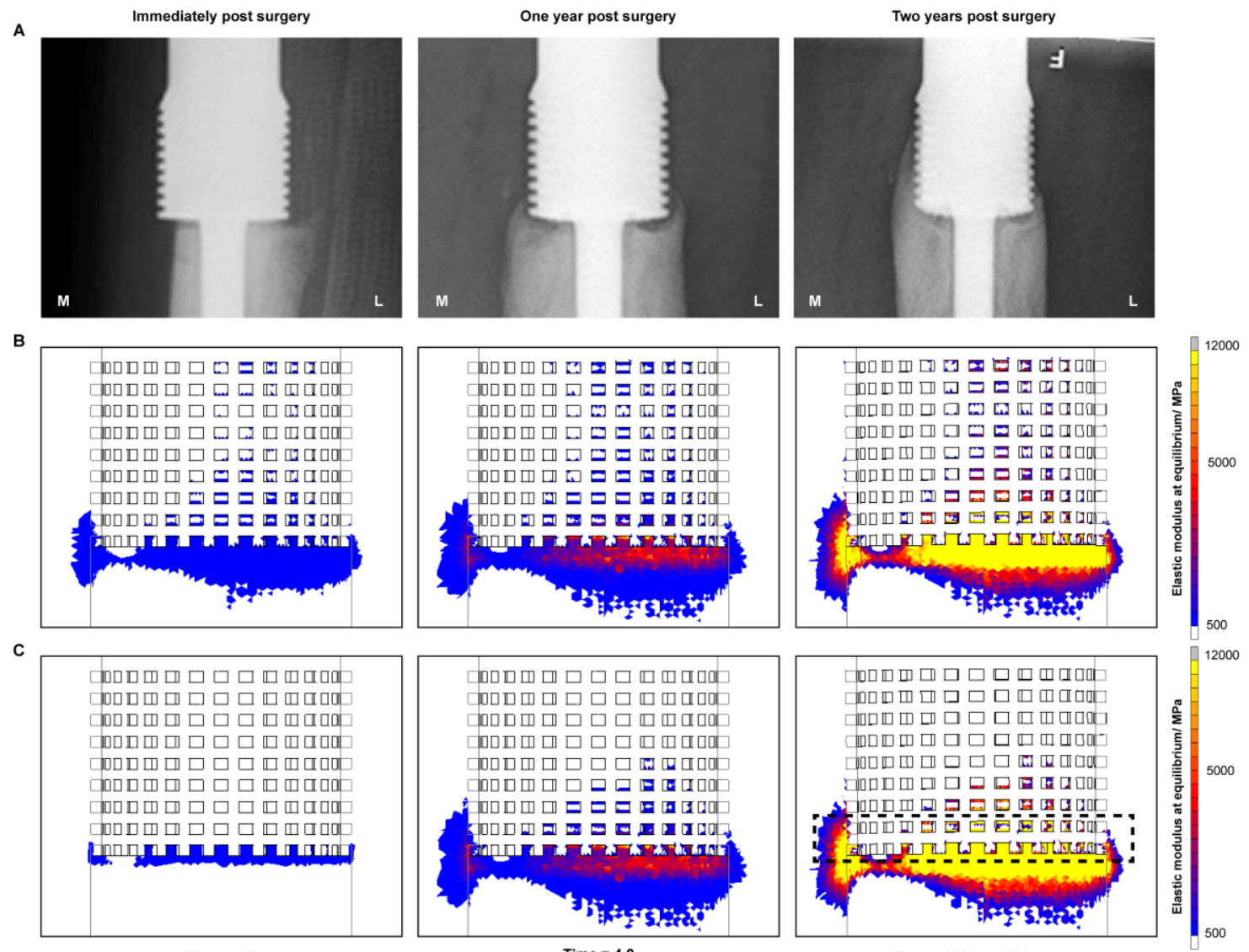

D

Time $=0.2$

Time $=4.0$

Time $=150$ (equilibirum)

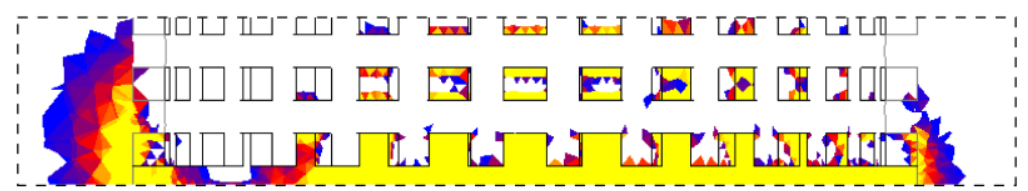

Fig. 7: Comparison of (A) follow-up radiographs and bone remodelling predicted for soft tissue envelope around grooved collar of segmental prosthesis at different time points; (B) Adaptive elasticity model; (C) Osteoconnectivity model; (D) Zoom of lower layers of osteoconnectivity model (indicated by dashed box in C), time $=150$ (equilibrium). Some remodelling occurred at the lower end of the tissue scaffold (not shown). Scale shows elastic modulus. 